\title{
ENHANGEMENT OF GHEMICAL CONSTITUENTS IN HYDROSOL AND RESIDUAL WATER OF AQUILARIA MALACCENSIS TETRAPLOID
}

\author{
Siti-Suhaila $\mathrm{AR}^{1}$ *, Mohd-Saleh $\mathbf{N}^{2}$, Norwati $\mathbf{M}^{1}$, Namasivayam $\mathbf{P}^{3}$, Mahani $\mathbf{C}^{4} \&$ Kandasamy $\mathbf{K I}^{5}$ \\ ${ }^{1}$ Forest Research Institute Malaysia, 52109 Kepong, Selangor, Malaysia \\ ${ }^{2}$ Agro-Biotechnology Institute, National Institute of Biotechnology Malaysia, 43400 Serdang, Selangor, Malaysia \\ ${ }^{3}$ Faculty of Biotechnology and Biomolecular, Universiti Putra Malaysia, 43300 Serdang, Selangor, Malaysia \\ ${ }^{4}$ Faculty of Science and Technology, Universiti Kebangsaan Malaysia, 43600 Serdang, Selangor, Malaysia \\ ${ }^{5}$ BioEconomy Corporation (M) Sdn Bhd, 61800 Kuala Lumpur, Malaysia \\ *sitisuhaila@frim.gov.my
}

Submitted May 2019; accepted October 2019

\begin{abstract}
Polyploidisation is an important feature of species evolution in plant, animal and fungal kingdoms. Many researches proved polyploid organisms outperform their diploid relatives in several aspects. Thus, many plant breeders in the last century practised induced and/or used natural polyploids to obtain improved plant cultivars. Fresh samples of A. malaccensis tetraploid contain higher amount of important agarwood sesquiterpenes compared with diploid counterparts. In this study, an investigation on the presence of important agarwood sesuiterpenes in hydrosol and residual water in diploid and tetraploid A. malaccensis was carried out. Three controls were used, namely, diploid seedlings grown in soil, diploid seedlings grown under control conditions (in vitro) and tissue culture-derived shoots of diploid for comparison with tetraploid A. malaccensis. Results showed that hydrosol water from tetraploid leaf and root samples contained the highest amount of important agarwood sesquiterpenes, i.e. 30.92 and $36.83 \%$ respectively. In residual water, the tetraploid $A$. malaccensis contained the highest amount of important agarwood sesquiterpenes in root sample $(49.1 \%)$. This article showed that polyploidy can be used as a tool to enhance the presence of important agarwood sesquiterpenes in A. malaccensis.
\end{abstract}

Keywords: Agarwood, sesquiterpenes, diploid, polyploids, compounds

\section{INTRODUCTION}

Aquilaria malaccensis (Thymealaeceae) is one of 25 Aquilaria species (agarwood-producing species) distributed throughout Peninsular Malaysia, Indonesia, India, Bangladesh and Thailand (Tajuddin \& Yusoff 2010, Nor-Azah et al. 2013). It is considered as one of the most expensive natural products existing today and is known among agarwood traders as the 'black gold of the forest' used to make valuable products such as pure agarwood oil, cosmetics, medicines and incense (Nor-Azah et al. 2013). How agarwood is formed is not yet fully understood, but the general understanding is that it is formed and permeates the heartwood as a stress response to wounding, microbial infection or both (Nor-Azah et al. 2013, Siti-Suhaila et al. 2015). Sesquiterpenoids and phenylethyl chromone derivatives are the principal compounds found in the agarwood oil, with $\alpha$-guaiene, $\delta$-guaiene and $\alpha$-humulene making up the major volatile compounds (Nor-Azah et al. 2013). In addition to agarwood essential oil, the water-soluble fraction called hydrosol (also known as floral water) is a valuable by-product obtained by hydrodistillation. Hydrosol contains all the agarwood essential oils in low concentrations, making it suitable for making products where pure essential oil would be considered too strong. Agarwood-based products such as soap, perfume water and drinking water usually incorporate hydrosol water and residual water. As wild $A$. malaccensis populations in natural forests shrink and agarwood demand increases, nonconventional methods in agarwood production need to be explored.

Polyploidisation or chromosome doubling is known to have an effect on many physiological properties of a plant, e.g. producing larger reproductive and vegetative plant parts (Mishra et al. 2010, Dhooghe et al. 2011) and enhancing 
secondary metabolite production (Gonzalez \& Weathers, 2003, Lavania 1988). Plants such as Artemisia annua, Vetiver and Papaver species exhibited increased key secondary metabolite content through polyploidisation (Hyder et al. 2002, Dhooghe et al. 2011, Ismail et al. 2013). Enhanced content of artemisinin, a secondary metabolite in A. annua, was achieved by polyploidisation in its hairy roots. Datura and Papaver plant species showed very high (almost $100 \%$ ) increases in secondary metabolites after chromosome doubling (Lavania 1988, Dhooghe et al. 2011). The present study was conducted to identify and compare compounds present in hydrosol and residual water by-products of diploid and polyploid A. malaccensis to conclude which plant was better quantitatively (number of chemical constituents detected) and qualitatively (number and amount of key chemical constituents) between the two ploidy levels.

\section{MATERIALS AND METHODS}

\section{Plant materials}

Four-month old tetraploid plantlets (TC) were compared with three types of control (similar age): soil-grown diploid seedlings (DS), in vitrogrown seedlings (DV), and in vitro-grown tissue culture-derived plantlets (DC). Plant parts (leaf, stem and roots) were separated and subjected to hydrodistillation processes.

\section{Chemical constituents analysis}

Plant parts (leaf, stem and root) from all plant samples were weighed at $200 \mathrm{~g}$ and placed into individual round-bottom flasks containing $1 \mathrm{~L}$ of distilled water. The samples were boiled for 6 hours. Then, at the end of the process, hydrosol and residual water were collected for further analysis. Hydrosol is water condensed in the Clavenger apparatus and residual water refers to water that is left in the flask. The liquid form samples were subjected to HS-SPME followed by GC-MS analysis (Nor Azah et al. 2016, Abd-Majid et al. 2017). The temperature programme was set initially at $60{ }^{\circ} \mathrm{C}$ for $10 \mathrm{~min}$ and increased to $230{ }^{\circ} \mathrm{C}$ at $3{ }^{\circ} \mathrm{C} \mathrm{min}-1$. The final temperature was held for $10 \mathrm{~min}$. The chemical constituents was identified by comparing their mass spectrum with database WileyNist05.L and HPCH2205.L.

\section{Statistical analyses}

Mean percentage values (average \pm SD) were used to determine the compounds present in the samples of hydrosol water and residual water from tetraploid and diploid A. malaccensis. Peaks were identified by comparing the retention times of the samples with those from the National Institute of Standards and Technology database.

\section{RESULTS AND DISCUSSION}

\section{Analyses of hydrosol}

Hydrosol from TC leaf samples showed the highest amount of sesquiterpenes $(50.64 \%)$ than all three controls (Table 1). Controls, namely, DS, DV and DC contained 42.68, 8.49 and $11.91 \%$ sesquiterpenes respectively. Furthermore, $61.06 \%$ of detected sesquiterpenes in TC were important (i.e. key chemical constituents) agarwood sesquiterpenes. Eudesmol is usually found in high quality agarwood essential oil (Tajuddin \& Yusoff, 2010, Nor-Azah et al. 2013) and it was found very high in TC leaf samples. DC samples had the second highest amount of important agarwood sesquiterpenes $(20.57 \%)$ after tetraploid followed by DS $(2.39 \%)$. No important agarwood sesquiterpenes were detected in DV. The differences in the amount of sesquiterpenes detected could be due to several factors. Firstly, the conditions where the plants were grown were different. Even though the starting materials, seeds for example, were the same, growing the seeds in soil under normal conditions could develop different types and/ or amount of sesquiterpenes than those grown in vitro. Secondly, it could due to the functions of the sesquiterpenes produced whereby they were different in terms of chemical profiling and/or amount of sesquiterpenes. The fact that DV samples produced the least amount of total sesquiterpenes $(8.49 \%)$ was an indication that the sesquiterpenes produced at an earlier stage might be used for defense mechanisms towards pathogens compared with the rest of the samples. This could explain why samples from DC produced less sesquiterpenes, i.e. almost the same amount produced by DV, because less or no pathogen existed in in vitro culture. Thirdly, the differences in the amount of sesquiterpenes produced might be due to different ploidy level, whereby tetraploid produced the highest amount 


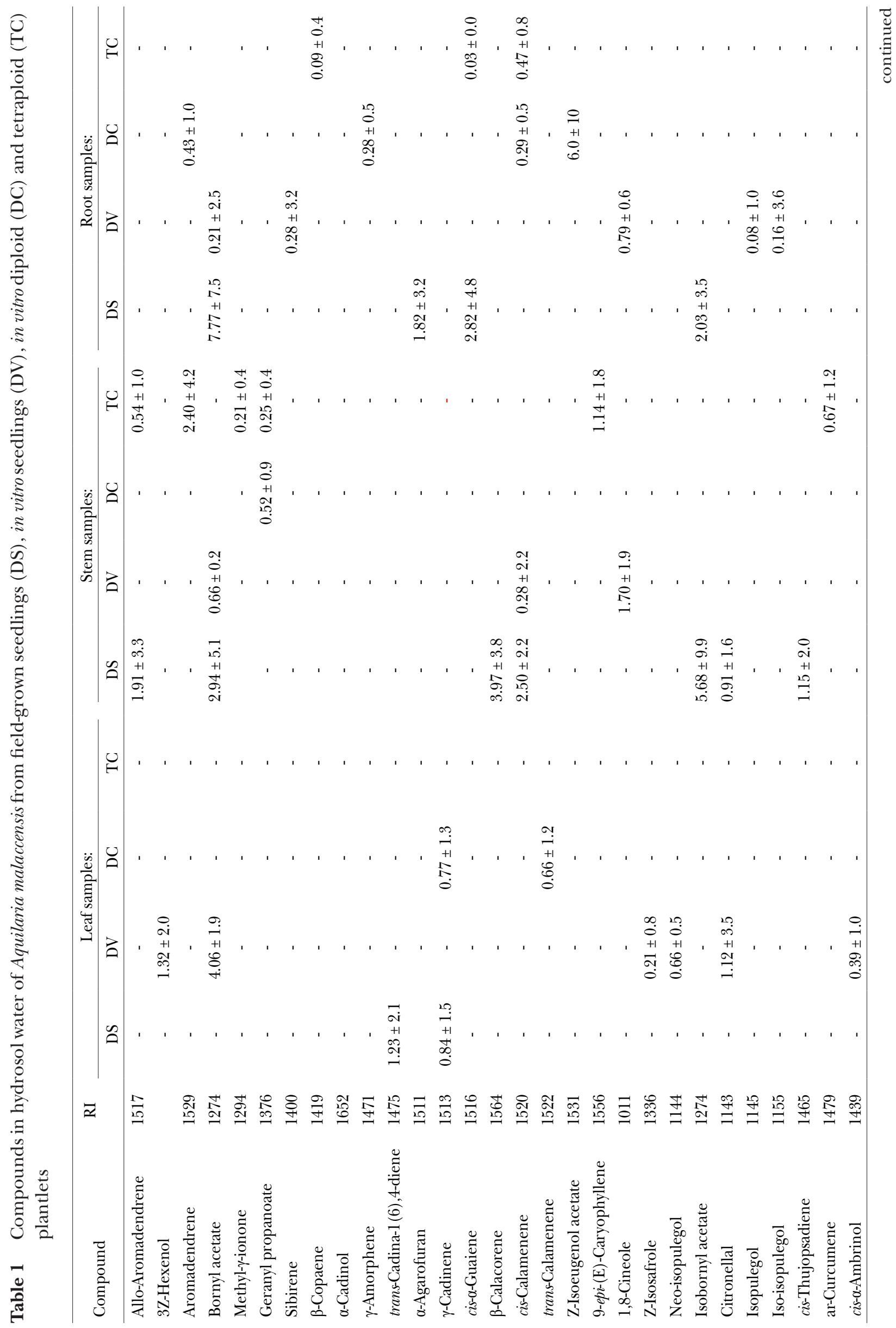




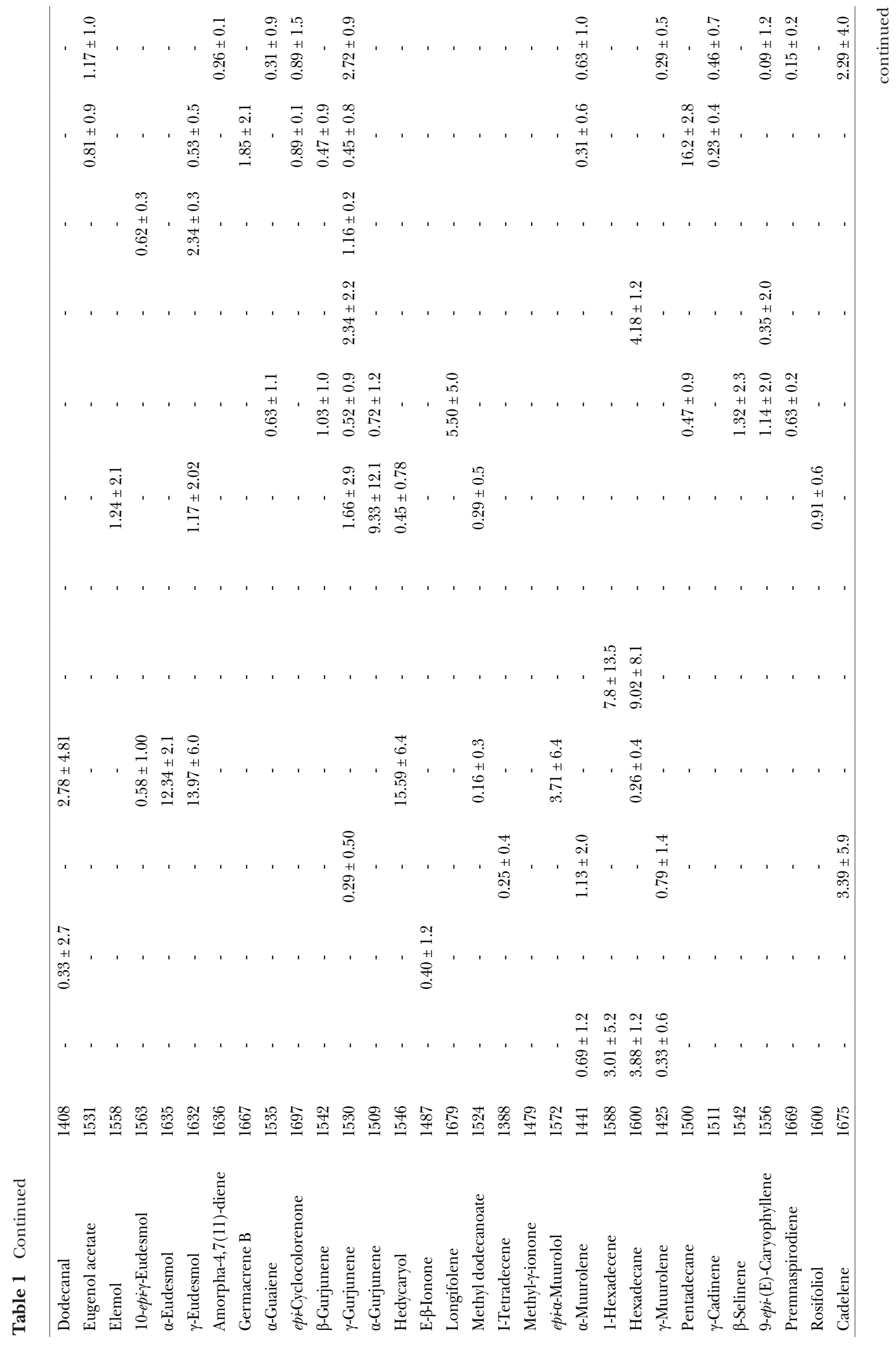




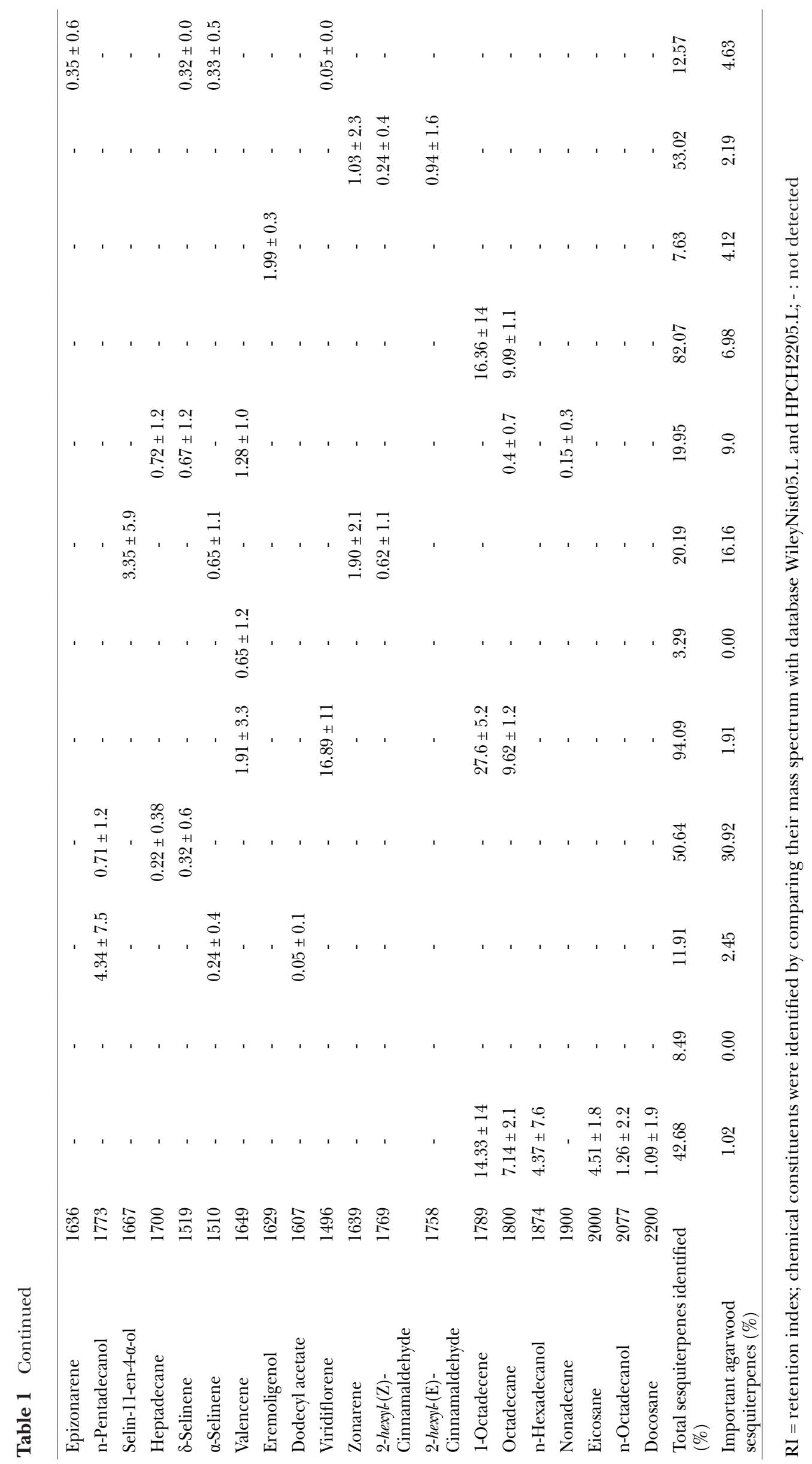


of total sesquiterpenes and the higher amount of important agarwood sesquiterpenes could be due to polyploidisation, i.e. chromosome doubling effect. Other researches also mentioned of the effects polyploidisation towards secondary metabolites (sesquiterpenes) produced were higher compared with its diploid counterparts.

In the stem samples, sample from DS had the highest amount of total sesquiterpenes $(94.09 \%)$ followed by DC $(20.19 \%)$, TC $(19.95 \%)$ and DV $(3.29 \%$ ) samples (Table 1). However, DS stems contained very low amount of important agarwood sesquiterpenes $(1.06 \%)$. The highest amount of important agarwood sesquiterpenes was obtained from DC ( $80 \%)$ followed by TC $(45.11 \%)$. Seedlings grown in soil produced the highest amount of sesquiterpenes, possibly for defense mechanism as very high amount was found in hydrosol water of DS. In root samples, the highest amount of sesquiterpenes detected was from DS $(82.07 \%)$, followed by DC $(53 \%)$, TC $(12.57 \%)$ and lastly from DV $(7.63 \%)$. However, of this, important agarwood sesquiterpenes detected was only $8.5 \%$. Important sesquiterpenes was higher in TC $(36.83 \%)$ and DV $(13.11 \%)$. The least amount of important agarwood sesquiterpenes was detected in DC $(1.87 \%)$. This could be due to the fact that most of the sesquiterpenes produced by DS are for defense mechanism, and less was required from other samples due to in vitro growing conditions.

\section{Analyses of residual water}

The residual water sesquiterpenes were only detected from DV and TC (Table 2), with higher levels found in the leaf of DV $(49.98 \%$ of sesquiterpenes), of which $14.31 \%$ were important agarwood sesquiterpenes. DC samples contained $19.63 \%$ total sesquiterpenes and only $3.45 \%$ was important agarwood sesquiterpenes. In stem samples, a total peak area of $5.90 \%$ was detected in DV and $1.83 \%$ in TC. Unfortunately, none of the sesquiterpenes were important agarwood sesquiterpenes. Among the root tissue samples, DV contained a higher amount of sesquiterpenes $(17 \%)$ than TC $(3.95 \%)$. However, TC root samples contained high amount of important agarwood sesquiterpenes $(49.1 \%)$ while they were detected in DV root samples. The low or undetectable amounts of compounds in residual water indicated that most of the chemicals were released during hydrodistillation. Higher levels of sesquiterpenes detected in TC compared with diploid plants in the present study, may be due to polyploidisation which enhances secondary metabolite production in plants (Lavania 1988, Chakrabarty et al. 1994, Dhooghe et al. 2011). Another major factor influencing secondary metabolite production is growth condition. Seedlings grown under natural conditions contain high levels of total volatile compounds compared with plant tissues grown under control conditions (in vitro) (Achakzai et al. 2009, Nikolova et al. 2013). Production of secondary metabolites can also increase in higher light intensity whereby many such compounds are important in plant defense and produced in response to external threat stimuli (Mole \& Waterman 1987, Mole et al. 1988). Therefore, lower levels may be produced in in vitro plants that are unlikely to be attacked or damaged by biotic and/or abiotic factors.

The in vitro tetraploids in our study produced high levels of important sesquiterpenes, indicating higher gene expression levels in the tetraploid than in the diploid plants. The large quantities of important compounds detected in the hydrosol from all plant samples showed that hydrodistillation can efficiently extract compounds from A. malaccensis. The higher yields of secondary metabolites by the tetraploid A. malaccensis in our study are consistent with those reported for the tetraploid rose (Rosa damascene), which is widely used to produce rose oil and rose water, and is probably triparental in origin (Iwata et al. 2000, Yokoya et al. 2000). Our results indicated that while the total quantity of compounds was about the same in diploid and tetraploid A. malaccensis, the composition of these compounds was of better quality in tetraploids, which had relatively higher levels of important sesquiterpenes.

Cells with higher ploidy levels have larger genome sizes, and are expected to have higher levels of transcription than cells with lower ploidy levels (Albuzio et al. 1978, Lavania 1988, Dhawan \& Lavania 1996). Therefore, gross expression of most or some of these genes would be expected to increase linearly with ploidy level. Previous studies have demonstrated that different parts of plants produce different secondary metabolites (Springer et al. 2002, Mishra et al. 2010, Nikolova et al. 2013) and the results of our study are in line with these different compounds detected in the leaves, stems and roots of diploid and tetraploid 


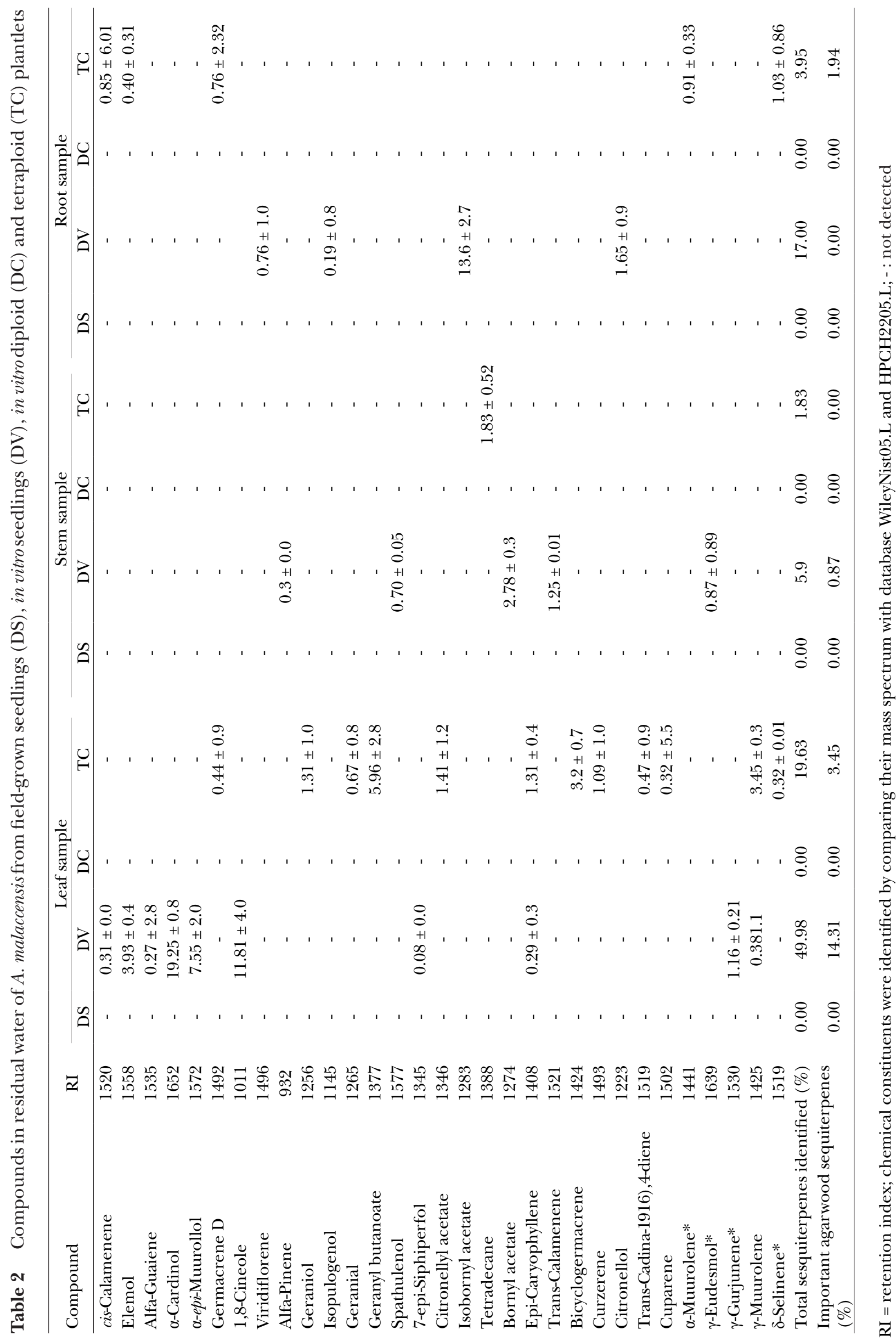


A. malaccensis. The enhanced production of important compounds in in vitro tetraploids can potentially add more value to $A$. malaccensis than its diploid counterparts.

\section{ACKNOWLEDGEMENTS}

The authors thank FRIM for financial support under the Tenth Malaysia Plan (Vote 10310802005) and Universiti Kebangsaan Malaysia for use of experimental facilities.

\section{REFERENGES}

Abd-Majid J, Hazandy AH, Paridah MT et al. 2018. Determination of agarwood volatile compounds from selected Aquilaria species plantation extracted by Headspace-Solid Phase Microextraction (HS-SPME) method. The Wood and Biofiber International Conference (WOBIC 2017). 21-23 November 2017, Selangor. IOP Conference Series: Materials Science and Engineering 368. 012023. doi:10.1088/1757899X/368/1/012023.

Achakzai AKK, Achakzai P, Masood A, Kayani SA \& Tareen RB. 2009. Response of plant parts and age on the distribution of secondary metabolites on plants found in Quetta. Pakistan Journal of Botany 41: 2129-2135.

Albuzio A, Spettoli S \& Cacco G. 1978. Changes in gene expression from diploid to autotetraploid status of Lycopersicon esculentum. Physiologia Plantarum 44: $77-80$.

Chakrabarty K Kuner A \& Manon V. 1994. Trade in Agarwood. WWF-India TRAFFIC India, New Delhi.

Dhawan OP \& Lavania UC. 1996. Enhancing the productivity of secondary metabolites via induced polyploidy: a review. Euphytica 87: 81-89.

Dhooghe E, Van Laere K, Eeckhaut T, Leus L \& Van HuylenBroeck J. 2011. Mitotic chromosome doubling of plant tissues in vitro. Plant Cell Tissue Organ Culture 104: 359-373.

Gonzalez DJ \& Wheathers PJ. 2003. Tetraploid Artemisia annua hairy roots produce more artemisinin than diploids. Plant Cell Reports 21: 809-813.

Hyder PW, Fredrickson EL, Estell RE, Tallez M \& Gibbens RP. 2002. Distribution and connection of total phenolics, condensed tannins, and nordihydroguaiaretic acid (NDGA) I creosotebush (Larrea tridentate). Biochemical Systematic and Ecological 30: 905-912.

Ismail N, Mohd-Ali NA, Jamil M, Mohd-Hezri FR, Tajuddin SN \& Mohd-NasiR T. 2013. Analysis of high quality agarwood oil chemical compounds by means of SPME/GCMS and Z-Score Technique. Malaysian Journal of Analytical Sciences 17: 403-413.

Inata H, Kato T \& Ohno S. 2000. Triparental origin of Damask roses. Gene 259: 53-59.

LAVANIA UC. 1988. Enhanced productivity of the essential oil in the artificial autopolyploid of vetiver (Vetiveria zizanioides L. Nash). Euphytica 38: 271-276.

Mishra BK, Pathak S, Sharma A, Trivedi PK \& Shukla S. 2010. Modulated gene expression in newly synthesized auto-tetraploid of Papaver somniferum L. South Africa Journal of Botany 76: 447-452.

Mole S \& Waterman PG. 1987. Tannins as anti feedants to mammalian herbivores: still an open question? Pp 572-587 in Waller GR (ed) Allelochemicals: Role in Agriculture and Forestry. American Chemical Society, Washington. doi: 10.1021/bk-1987-0330.ch051.

Mole S, Ross JAM \& Waterman PG. 1988. Light induced variation in phenolic levels in foliage of rain-forest plants. Journal of Chemical Ecology 14: 1-21.

Nikolova M, Petrova M, Zayona E, Vitkova A \& Evstatieva L. 2013. Comparative study of in vitro, seedlings and in vivo grown plants of Arnica montana-polyphenols and free radical scavenging activity. Acta Botany Croatia 72: 13-22.

Nor-Azah MA, Mailina J, Abd-Majid J et al. 2016. Chemical composition of Litsea cubeba essential oil from Cameron Highlands. Pp 128-132 in Chee BJ et al. Proceedings of 14th Seminar on Medicinal E० Aromatic Plants Healing Power from Nature: Current Trends $\mathcal{E}^{\circ}$ Perspectives. FRIM Proceedings No. 8. 11-12 October 2016. Forest Research Institute Malaysia, Kepong.

Nor-Azah MA, Nurlaila I, Mailina J, Mohd-Nasir T \& MohDHEzRI FR. 2013. Analysis of aroma compounds from gaharu oil by headspace solid phase microextraction and gas chromatography-mass spectrometry. Pp180-185 in Seminar on Medicinal E Aromatic Plants Proceedings. 25-26 September 2013, Kuala Lumpur.

Siti-Suhaila AR, Mohd-Saleh N, Norwati M et al. 2015. Polyploid induction in native Malaysian agarwoodtoward the development of improved breeding stock of Aquilaria malaccensis. Journal of Tropical Forest Science 27: 376-387.

Springer TL, Mcgraw RL \& Aiken GE. 2002. Variation of condensed tannins in roundhead Lespedeza germplasm. Crop Sciences 42: 2157-2160.

TAjuddin SN \& Yusoff MM. 2010. Chemical composition of volatile oils of Aquilaria malaccensis (Thymeleaeceae) from Malaysia. Natural Product Communications 5: 1965-1968.

Yokoya K, Roberts AV, Mottley J, Lewis R \& Brandham PE. 2000. Nuclear DNA amounts in roses. Annal Botany 85: 557-561. 